\title{
Staatsnorm und Kongo-Intervention: Die Herstellung gescheiterter Staatlichkeit unter Interventionspersonal
}

\author{
Kai Koddenbrock
}

\section{Einleitung}

Internationale Versuche, den kongolesischen Staat zu stärken, benötigen ein klares Verständnis von zweierlei: dem Staat und der Demokratischen Republik Kongo. ${ }^{1}$ Statebuilding muss wissen, was genau es bauen will. Dieses doppelte Erfordernis wird jedoch auf überraschende Weise eingelöst. So bezweifeln manche Analysen die Existenz des Kongos rundheraus: "Sie nennen es ein Land, tatsächlich ist es nur ein Zaire-förmiges Loch in der Mitte Afrikas" (Economist 1998) oder »Die Demokratische Republik Kongo existiert nicht" (Herbst/Mills 2009) sind zwei prominente Beispiele für diese Praxis. Rhetorische Aufhänger orientieren sich an den Erwartungen des Publikums. Für dieses Publikum scheint es fraglich zu sein, ob der Kongo überhaupt existiert.

Der souveräne Staat und das anarchisch oder hierarchisch strukturierte Staatensystem hingegen sind lange das konzeptionelle Kernstück der Internationalen Beziehungen (IB) gewesen. Souveränität und Staat wurden nicht nur selbstverständlich vorausgesetzt, sondern fungierten auch als zentrale Normen internationaler politischer Praxis. Politische Kollektive mussten Staaten werden, um auf der internationalen Bühne ernst genommen zu werden. Diese Sollens-Funktion des Staates zeigt seinen NormCharakter. Zugleich stellt der Staat schon immer eine der schwierigsten Herausforderungen für die politische Soziologie dar. Was in der internationalen politischen Praxis selbstverständlich ist, ist höchst umstritten in der akademischen Reflexion.

Wenn der Kongo nicht als inexistent angesehen wird, wird er zumeist als schwacher oder gescheiterter Staat gefasst. Die IB braucht den Staat,

1 Im Folgenden benutze ich aus Gründen der Lesbarkeit ,Kongo“ für die Demokratische Republik Kongo, obwohl die kolonialen Konnotationen in dieser Wortwahl ausgeprägt sind. Die argumentative Stoßrichtung des Aufsatzes sollte jedoch deutlich machen, dass mir an einer Problematisierung dieser post-kolonialen Wissensordnung gelegen ist. 
während der gescheiterte Staat förmlich am Kongo haftet, wenn er in Beratungspapieren, akademischen Publikationen und Hilfspraktiken konzeptionalisiert wird. Diese Symmetrie soll im folgenden Aufsatz untersucht werden. Mit Hilfe jüngerer staatstheoretischer Konzepte werde ich argumentieren, dass der Staat als performativer Effekt sozialer Praktiken verstanden werden kann, der immer wieder hergestellt werden muss. Gleichzeitig ist der Staat eine einflussreiche Norm. Der Beitrag untersucht also die performative Wirkung der Staatlichkeitsnorm und der Praktiken der Zuschreibung von gescheiterter Staatlichkeit im Rahmen des Statebuildings. In einem zweiten analytischen Schritt werde ich auf Basis von semistrukturierten, qualitativen Interviews in Goma im Ostkongo und in New York ein Gedankenexperiment unternehmen, über die Staatsnorm hinauszudenken, denn Intervenierende im Kongo fassen den Kongo sowohl als gescheiterten Staat als auch als etwas Anderes, dessen Inhalt genauer zu bestimmen ist.

Sich der Staatsnorm zu entledigen, schafft Raum dafür, über alternative Antworten auf globale Herausforderungen nachzudenken und ermöglicht einen Blick darauf, dass nicht alles gescheitert ist in diesen scheinbar gescheiterten Staaten. Rationalität und zielgerichtetes Verhalten existieren auch im Kongo. Dieser Beitrag interveniert also in die metatheoretische Debatte um das Verhältnis von Norm, Diskurs und Praktik (vgl. Beiträge von Graf, Glaab und Engelkamp; Renner sowie Jacobi und Kuntz), die in diesem Sammelband verfolgt wird, und versucht sich zugleich an einer Kritik der Staatsnorm und ihrer Konsequenzen für die Selbstverständlichkeit von Intervention (siehe auch Koddenbrock 2016). Der Aufsatz versteht sich also ein Beitrag zur ,kritischen Normenforschung', wie sie jüngst in der ZIB eingefordert wurde (Engelkamp et al. 2012) und in diesem Band weiterverfolgt wird. ${ }^{2}$

\section{Konzeptionen des (gescheiterten) Staates}

Aktuelle Probleme mit dem Konzept des gescheiterten Staates werden im Völkerrecht seit mehr als einem Jahrhundert diskutiert: Ist der Staat ein faktisches Machtzentrum oder eine soziale Konstruktion oder sogar beides? (vgl. Koskenniemi 2005: 232). Carl Schmitts Antwort auf diese Frage lautete, dass die Souveränität darin liege, über den Ausnahmezustand zu ent-

2 Zur Notwendigkeit, praxistheoretische Analysen auch materialistisch zu fundieren und dessen Bedeutung für den Kritikbegriff siehe Koddenbrock 2015.. 
scheiden. Martti Koskenniemi (2005) nennt dies den pure-fact approach. Aus dieser Perspektive entsteht Souveränität aus der Macht und Fähigkeit zur Entscheidung. Daraus folgt, dass Staatsbildung kein legaler oder konzeptueller Prozess ist, sondern einer von Machtkämpfen. Staaten beginnen zu existieren, wenn ein Kollektiv die Fähigkeit zur Selbststeuerung erlangt hat (Koskenniemi 2005: 272). Da diese Fähigkeit jedoch von anderen anerkannt werden muss, um wirkmächtig zu werden, stellt sich unmittelbar die Frage nach den Kriterien, anhand derer diese Fähigkeit evaluiert werden kann. Diese Kriterien sind nun zwingend dem Staat vorgängig und fuBen auf einem "normativen Code « (Koskenniemi 2005: 274), der sowohl der Existenz der Souveränität als auch des Staates vorausgeht. Im Internationalen Recht oszilliert der Staat somit von Beginn an zwischen einem/ einer AkteurIn mit Fähigkeiten und einem normativen Konzept.

Trotz dieser Debatten im Völkerrecht und der Arbeiten poststrukturalistischer IB, die wiederholt die Staatsidee in Frage stellten (Ashley 1988; Campbell 1998; Weber 1998), fällt die Policy-Debatte immer wieder auf den pure-fact approach zurück, wenn es um sogenannte gescheiterte Staaten geht. Die Policy-Debatte im Bereich der Konfliktforschung, aber auch der Entwicklungspolitik, dreht sich aktuell zentral um die Frage der Institutionen und im gleichen Atemzug auch um die Staatlichkeit und ihr Scheitern (Paris 2004; Chandler 2010: 65-93; Autesserre 2012; Weltbank 2011; Koddenbrock 2016). Schwache Staatlichkeit ist zu einem buzzword in der westlichen Entwicklungspolitik geworden und der Staat wird wieder zum zentralen Akteur (Deutsches Institut für Entwicklungspolitik 2009). Jüngste OECD- und Weltbank-Berichte zeigen überdies, wie sehr die Idee des Staatsversagens mit einer funktionalistischen Perspektive auf den Staat als eine Art Akteur mit bestimmten Fähigkeiten verbunden ist und damit auf dem pure-fact approach beruht. Für die OECD hat ein schwacher Staat eine »weak capacity to carry out basic functions of governing a population and its territory, and lacks the ability to develop mutually constructive and reinforcing relations with society (OECD 2011: 21). Der World Development Report 2011 der Weltbank definiert fragility als "periods when states or institutions lack the capacity, accountability, or legitimacy to mediate relations between citizen groups and between citizens and the state, making them vulnerable to violence« (Weltbank 2011: xvi). Diese beiden Auffassungen belegen, dass der Staat aktuell als Akteur mit bestimmten Fähigkeiten konzeptualisiert wird.

Die politische Soziologie und Anthropologie des Staates debattierte in den letzten Jahrzehnten immer wieder die "difficulty of studying the state" (Abrams 1988), indem die Komplexität von Staatsbildungsprozessen (Wilke und Schlichte 2000; Bliesemann de Guevara, 2012) untersucht wurde, 
aber immer auch die Unumgänglichkeit des Staates in der Untersuchung von Herrschaft unterstrichten wurde (Hansen und Stepputat 2001: 8). Aufgrund der konstituierenden Rolle des souveränen Staates für das internationale System setzte die IB lange den Staat in besonderem Maß unhinterfragt voraus. In den letzten 30 Jahren wurde diese zentrale Rolle des Staates als vorgängige und abgeschlossene Einheit globaler Politik jedoch kritisiert. Regime (Keohane 1985) und Normen (Finnemore/Sikkink 1998) rückten ebenso in den Blick wie internationale Organisationen als eigenständige Akteure (Barnett/Finnemore 2004).

Governance und governmentality sind heute zwei der zentralen Versuche, konzeptionelle Zugänge zur globalen Politik zu finden, die den Staat nicht als gegeben voraussetzen und damit performativ stützen (Sending/ Neumann 2006). Das Wachstum der EU-Forschung mit Begriffen wie multi-level governance hat ebenfalls zu Analysen beigetragen, die den Staat nicht mehr als ausschließliches Zentrum der Analyse setzen. Der sogenannte practice turn in den IB (Neumann 2002; Adler/Pouliot 2011; Bueger und Gadinger 2014; vgl. auch Graf, Glaab und Engelkamp in diesem Band) trägt zu dieser IB jenseits des Staates bei, denn Praktiken können von allen Arten von Akteuren vollzogen werden und stellen somit eine Abkehr vom Staatszentrismus dar.

Trotz dieser theoretischen Absetzbewegungen ist die Staatsnorm weiterhin äußerst einflussreich. Der gescheiterte Staat hat eine besondere Beziehung zur Staatsnorm, weil er diese Norm stabilisiert, indem er ihre Nichtbefolgung oder -umsetzung postuliert. Ein gescheiterter Staat befindet sich konzeptionell in einer schwierigen Situation: Er ist kein Staat, da er gescheitert ist. Er ist jedoch trotz seiner Schwächen ein gescheiterter Staat. Die Wahl dieser Terminologie für eine soziale Situation, in der Analysen die Abwesenheit des Staates konstatieren, zeigt, wie einflussreich die Staatsnorm bleibt. Sogar Nicht-Staaten werden Staaten genannt.

Aus einer praxistheoretischen performativen Sicht betrachtet wird der Staat kontinuierlich hergestellt - durch Praktiken. Ich werde im Folgenden nach einem kurzen Einblick in die Debatten um Praxistheorie und performativity analysieren (vgl. hierzu auch den Beitrag von Jacobi und Kuntz in diesem Band), wie dieses Herstellen des Staates als gescheiterter Staat unter Interventionspersonal, das im und über den Kongo arbeitet, funktioniert. Intervenierende stellen einerseits den Kongo her, indem sie ihre Beobachtungen der sozialen Realität des Kongos im Lichte der Staatsnorm und dem resultierenden Konzept des gescheiterten Staates interpretieren. Andererseits stellen sie den Kongo auf gänzlich andere Weise her, wenn sie aus einer Vogelperspektive auf den Kongo blicken. Plötzlich spielt die Staatsnorm eine untergeordnete Rolle. Stattdessen wird der Kon- 
go begrifflich über die menschliche Natur, Wettbewerb und Zusammenarbeit oder die Rolle der Geschichte analysiert. Es wäre folglich vereinfachend, den Staat als schlicht selbst-verständlich unter Interventionspersonal zu analysieren. Manchmal ist er es, manchmal nicht. Der Stellenwert der Staatsnorm hängt somit auch von der Art und Weise ab, wie die Forscherin oder der Forscher den Staat schreiben (Weber 1990; vgl. auch Vrasti 2008).

Die folgende Analyse zeigt, dass die performative Herstellung des Kongos kein automatischer Prozess ist. Es gibt durchaus Wege, ihm zu entgehen und damit die Selbstverständlichkeit von westlicher Intervention und Statebuilding im Kongo zu unterlaufen. Die zwei unterschiedlichen Interpretationen, die ich unten anbiete, unterstreichen auch die zentrale Rolle des Forschenden in stärker ethnographisch arbeitenden Analysen des Staates. Wie die Interviewten perpetuiert auch die Analyse den Staat auf unterschiedliche Weise und kräftigt damit die Staatsnorm.

\section{Performative Praxis und der Staat}

Performativity in den IB hat zwei Quellen: Feministische IB, die sich weitgehend auf Judith Butlers Arbeiten zu sex und gender beruft (vgl. Weber 1998), und das wachsende Interesse an den science and technology studies und deren Auffassung von performativity (Mol 2002; Law/Urry 2004; Callon 2006; Barad 2007; MacKenzie et al. 2007). Aufbauend auf Butlers Interpretation von John Austins Sprechakttheorie (1962) sieht Cynthia Weber performativity als "the reiterative and citational practice by which discourse produces the effects that it names" (Weber 1998: 81). Auf Seiten der science and technology studies haben Michel Callon und Donald MacKenzie Studien dazu vorgelegt, wie die Ökonomie als Seinsbereich auch durch ökonomische Theorie oder Rechenmethoden hergestellt wird (Callon 1998; 2006; MacKenzie 2006). Sie lassen das representationalist idiom (Pickering 1994) hinter sich und argumentieren, dass man sich nicht auf die scheinbare Trennung von Realität und ihrer Repräsentation konzentrieren solle, sondern auf das, was getan werde. Dieses Tun wiederum könne erfolgreich sein oder scheitern.

»The success (or the failure) of an act of language becomes clear only at the end of the tests to which it is put, through the cooperation it triggers, the oppositions and controversies that it generates. Statements can survive and prosper in one particular place and at one particular 
time, and disappear in other places and at other times« (Callon 2006: 26).

Das Konzept des gescheiterten Staates beispielsweise ist sehr erfolgreich in der Politikwissenschaft. In der Anthropologie hingegen ist es seltener, möglicherweise weil die Anthropologie es als Teil ihres Selbstverständnisses betrachtet, kontextsensible und weniger universalistische Analysen der Ordnungsbildung weltweit vorzulegen. Das Konzept des gescheiterten Staats gedeiht also innerhalb des Netzwerkes der Politikwissenschaft bestehend aus Geldgebern, Personal, Zeitschriften, Statistiken etc., nicht jedoch in der Anthropologie (siehe auch Bueger/Bethke 2014).

Mithilfe von Callons oben stehendem Vorschlag, dass das Tun auch die Benutzung von Konzepten impliziert, nehme ich also an, dass der Kongo als gescheiterter Staat performativ hergestellt wird, indem das Konzept des gescheiterten Staates benutzt und in Zirkulation gehalten wird. Um meinen Zugang zum Staat als Effekt und Norm klarer zu machen, beziehe ich mich auf Michel Foucaults Vorschlag, den Staat einerseits als Effekt der analytischen Praktik zu verstehen, den Staat in soziale Prozesse hineinzulesen, und gleichzeitig als normativ vorzuziehende Form der politischen Organisation (Foucault 2006a: 416).

In seinen Vorlesungen zu Sicherheit, Territorium und Bevölkerung (Foucault 2006a) und zur Geburt der Biopolitik (Foucault 2006b) fasst Michel Foucault den Staat als performative Praktik:

»Der Staat ist also ein Verständnisprinzip einer schon bestehenden Wirklichkeit, einer schon bestehenden Gesamtheit von Institutionen... Zweitens fungiert der Staat innerhalb dieser politischen Vernunft als ein Ziel, d.h. als etwas, das als Resultat von aktiven Interventionen, das durch diese Vernunft, diese Rationalität erreicht werden soll.... Der Staat ist also ein Verständnisprinzip des Seienden, aber er ist auch etwas, das sein soll.« (Foucault 2006: 416).

Für Foucault ist der Staat aber nicht nur dies, sondern auch ein Effekt, der aus »tausend verschiedenen Vorgängen « (Foucault 2006: 347) hervorgegangen ist. Bringt man diese beiden Thesen zusammen, so liegt es nahe, einen - wenn auch nicht den einzigen ${ }^{3}$ - zentralen Vorgang, der zum Staats-Ef-

3 Materialistische Staatstheorie würde den Schwerpunkt auf soziale Kämpfe und hegemoniale Projekte legen. Marxistische IB versucht immer auch die Beziehung zwischen Staatsform und Kapitalismus im Blick zu behalten (siehe auch Koddenbrock 2015). 
fekt führt, in der Anwendung des Staates als Verständnisprinzip und Norm zu sehen. Damit ist der Staat auch ein Effekt performativer Praktik.

Um nun also die Herstellung des Kongos als gescheiterter Staat zu untersuchen, übernehme ich Foucaults Vorschlag, den Staat als Effekt zu betrachten, der aus der kontinuierlichen Praktik resultiert, ihn in die soziale Situation im Kongo hineinzulesen und zu schreiben. ${ }^{4}$ Aus dieser Sicht gibt es keine Dinglichkeit des Staates oder einen Staats-Akteur, an dem wir partizipieren können. Wir können nur an Praktiken teilnehmen, die den Staats-Effekt zur Folge haben. Aus der hier vertretenen Perspektive kann man an der Herstellung des Staates beteiligt sein, nicht aber am Staat selbst.

In einem einflussreichen Essay hat Timothy Mitchell diese Perspektive auf den Staat auf den Punkt gebracht:

»We should not ask 'Who is the state?' or 'Who dictates its policies?' Such questions presume what their answers pretend to prove: that some political subject, some who preexists and determines those multiple arrangements we call the state. The arrangements that produce the apparent separateness of the state create the abstract effect of agency, with concrete consequences." (Mitchell 1991: 90-91)

Mitchell schlägt also vor, den analytischen Fokus auf die »techniques that make the state appear to be a separate entity that somehow stands outside society" (Mitchell 1991: 91) zu verlagern.

Dieser Ansatz zieht eine Metaperspektive auf den Staat nach sich. Man könnte dies auch als eine Untersuchung der Möglichkeitsbedingungen des Staates bezeichnen. Aus dieser Perspektive sucht man nicht nach dem Staat an sich, sondern danach, wie seine Konstruktion und Trennung von der sozialen Welt sich technologisch und intellektuell vollzieht.

\section{Failed State Kritik und der Kongo}

Nach der ersten Welle von Analysen gescheiterter Staaten in den späten 1980ern und 1990ern (Jackson/Rosberg 1982; Helman/Ratner 1992; vgl. zur Kritik an diesen Ansätzen Bueger/Bethke 2014) entstand eine breite Kritik des Begriffs. Diese bezieht sich zumeist entweder auf die Unschärfe des Begriffs (Call 2011) und seine pathologisierenden Effekte (Bilgin/

4 Vgl. Kosmatopoulos (2011) für eine ähnliche Analyse in Bezug auf den Libanon und Heathershaw (2012) für grundsätzliche Überlegungen zu diesem Thema. 
Morton 2002; Bøås/Jennings 2006; Koddenbrock 2013). Alternativ betonen die Kritikerinnen und Kritiker die überraschende Resilienz des Staates, insbesondere dort, wo er eigentlich als gescheitert identifiziert wurde, oder sie fokussieren die Produktion politischer Ordnung außerhalb des Staates. Letztere Perspektive kritisiert das Konzept des gescheiterten Staats als übermäßig ideal-typisch und schlägt vor, die real governance an denjenigen Orten zu studieren, die häufig als Horte gescheiterter Staaten aufgefasst werden (de Olivier Sardan 2008; Titeca/de Herdt 2011; Renders/Terlinden 2010; Trefon 2009; Baaz/Olsson 2011).

Trotz ihrer Versuche, das Label des gescheiterten Staates zu nuancieren, beschäftigen sich diese Autorinnen und Autoren jedoch selten explizit mit dem Konzept des Staates. Pierre Englebert, eine einflussreiche Stimme in puncto kongolesischer Staat, schreibt zum Beispiel:

"Not only does Congo endure against all odds, but the Congolese even profess a remarkable fervor in their attachment to the state. Paradoxically, where one would expect to observe the formation or the rise to salience of alternative identities, one sees a nearly unanimous profession of loyalty to decrepit Congo, whether among government supporters, rebels or other opposition groups« (Englebert 2003: 4).

Englebert übersieht bei dieser Analyse, dass seine Quellen in diesem Fall von ihrer Liebe zum Kongo und zu seiner Einheit sprechen. Inwieweit sich diese Liebe zum Kongo mit ihrer Liebe zum Kongo als Staat deckt, bleibt völlig unklar. Der Kongo und der kongolesische Staat sind keine Äquivalente. ${ }^{5}$ In den einflussreichsten Werken zu den langen Jahren der Herrschaft Mobutus von 1965 bis 1997 wird der Staat als etwas aufgefasst, dass steigt und fällt (Young/Turner 1985) und das gemäß dem Modell der Familie organisiert wird (Schatzberg 1988: 83-98). Auch hier ist die Tendenz zu konstatieren, in Begriffen des Staates zu denken, obwohl es keine unmittelbaren logischen Zwänge dafür gibt.

5 Englebert zitiert den damaligen kongolesischen Präsident Joseph Kabila mit einer Aussage, in der er nicht über den Staat, sondern über die Nation spricht, um seine These von der Resilienz des kongolesischen Staates zu untermauern. In der zitierten Passage berichtet Kabila über seine Gespräche mit Oppositionsführern im Jahr 2002: »We have been able to get along on a [shared] nationalistic [and] patriotic basis that takes account of the superior interests of the nation « (Englebert 2003: 5). Es bleibt unklar, wo Englebert hier einen Beleg für die Bedeutung des Staates für Kabila entdeckt. 


\section{Zwei Kongos}

Mitchells Vorschlag, die Produktion des Effektes einer separaten Staatsentität zu untersuchen, verschafft uns ein wirksames Werkzeug, den Stellenwert der Staatsnorm in der Herstellung gescheiterter Staatlichkeit zu fassen. Wie Interventionspersonal kongolesische Politik analysiert wird nun zum Modus, durch den der Kongo zum gescheiterten Staat gemacht wird. Ich möchte jedoch noch einen Schritt weiter gehen. Anstatt nur zu zeigen, wie einflussreich die Staatsnorm ist und wie sehr sie das Denken in Begriffen gescheiterter Staatlichkeit perpetuiert, möchte ich die Debatte weiten und zeigen, dass es durchaus möglich ist, den Kongo nicht nur in Begriffen des Staates zu fassen. Weder ist die Staatsnorm unentrinnbar, noch ist es die ausdauernde Stabilisierung westlicher Interventionen in Orten, die ihrer scheinbar bedürfen.

Die Perpetuierung der Staatsnorm und die Herstellung der alternativen Vorstellung des Kongos finden an unterschiedlichen Orten, unter variierenden Akteuren und für vielfältige Publika statt. In diesem Kapitel konzentriere ich mich auf Interventionspersonal in Goma und New York. Eine Analyse von Beratungspapieren und Kongo-Expertisen habe ich andernorts vorgelegt (Koddenbrock 2012; 2014; 2016). Interventionspersonal in Goma und New York und kongolesische GesprächspartnerInnen in Goma brachten den Staat in den Interviews hervor, wenn sie direkt über ihre Einschätzung zur kongolesischen Politik gefragt wurden oder dazu, was denn der Kongo für sie sei. Als ich sie auf andere Weise zum Kongo gegen Ende des Interviews befragte, spielte der Staat keine Rolle. Sie legten das Staatskonzept nicht an das an, was sie als das Geschehen im Kongo präsentierten. Diese Erkenntnisse sollen dazu dienen, die Staatsnorm zu unterlaufen, ohne jedoch bereits bessere Alternative vorzustellen. Diese kritische Dekonstruktion der Staatsnorm dient dazu, die kontinuierliche Pathologisierung kongolesischer Politik zu unterminieren.

\subsection{Kongo als gescheiterter Staat}

Die folgende Analyse der Herstellung des Kongos als gescheiterter Staat fußt auf 66 Interviews, die ich in Goma und New York zwischen 2009 und 2011 führte. ${ }^{6}$ Ein Großteil des Materials resultiert aus den zwei Fragen

6 Siehe Koddenbrock 2016 für eine umfassendere Analyse der Interventionspraxis im Kongo. 
»Was ist der Kongo für sie?« und »Was denken Sie über kongolesische Politik? «Im Sinne der grounded theory kodierte ich die Antworten offen und stieß über mehrere Abstraktionsschritte hinweg zu den drei Modi der Verstaatung des Kongos vor, denen gemeinsam ist, dass sie den Kongo pathologisieren. Diese drei illustrativen Modi möchte ich Reifizierung, Gegenüberstellung und Kontextualisierung nennen. Reifizierung ist der am weitesten verbreitete Modus. Reifizierung bedeutet, dass der Staat schlicht vorausgesetzt und damit reifiziert wird. Dass im Kongo scheinbar kein Staat existiert, beweist dann sein Scheitern. In diesem Modus wird der Staat schlicht als natürliche und notwendige Form politischer Organisation angenommen und sein Fehlen im Kongo gesetzt. Der Modus der Gegenüberstellung funktioniert anders. Hier wird zunächst der ernsthafte Versuch unternommen, die sozialen und politischen Prozesse im Kongo analytisch und weniger wertend anzugehen. Plötzlich setzt sich jedoch der Staat als Norm und Verständnisprinzip durch und bewirkt einen erneuten failedstate-effect (Koddenbrock 2013). In dieser Gegenüberstellung fehlt die logische Verbindung zwischen der Beschreibung dessen, was im Kongo passiert, und dessen Pathologisierung als nicht-staatlich oder gescheitert-staatlich. Kontextualisierung bedeutet schließlich, dass die scheinbaren Defizite des kongolesischen Staates nicht schlicht behauptet, sondern in einen Kontext gesetzt werden. Es wird damit nicht angenommen, dass der Kongo vor allen Dingen aufgrund mangelnder Organisationsfähigkeit der Kongolesinnen und Kongolesen kein Staat ist, sondern erklärt dies vor allem mit materiellen, regional-globalen und auch historischen Umständen. Allen drei Modi ist gemein, dass sie den Kongo und seine sozio-politische Organisation pathologisieren, d.h. als prinzipiell interventionsbedürftig darstellen.

Zahlreiche Interviewte reifizieren und essentialisieren den Staat. Sie nehmen den Staat als natürliche Bedingung an, wenn sie Regierungspraxis im Kongo analysieren. Sie sind noch nicht fertig, wenn sie ihre Auffassung zur Regierung kundgetan haben, sondern zeigen ein starkes Bedürfnis, im Anschluss eine Aussage über den kongolesischen Staat zu treffen. Diese macht den Kongo schnell zu einem gescheiterten Staat. Der folgende Interviewausschnitt illustriert, wie der Staat als zentrales Verständnisprinzip für Regierungshandeln funktioniert. Langsame Entscheidungsfindung und parallele Kommandostrukturen werden als Indikatoren für einen schwachen Staat herangezogen, weil das Sein-Sollen des Staates dies auszuschlieBen scheint.

"The current situation is difficult. There has been some progress recently but obviously, until now, the expectations of the Congolese population were 
exaggerated and what the Congolese leaders achieved is without doubt even less than we hoped. The Congolese state remains extremely weak. The Congolese leaders take their time in making decisions especially with a view to security, which is essential to build state authority. We are in many areas incomplete..., although there is a Congolese army, at the moment there currently is no real Congolese army, there are men in, but it's also not a disciplined army in which there are several parallel chains of command. " 7

Heterogene Regierungspraktiken wie parallele Kommandostrukturen in der Armee zeigen für diesen EU-Offiziellen einen schwachen Staat an. Dass die kongolesische Führung in puncto Sicherheit Entscheidungen langsam fällt, wird sofort im Lichte seiner Bedeutung für den Aufbau staatlicher Autorität betrachtet. Dies impliziert, dass nur durch den Staat Sicherheit dauerhaft gewährleistet werden kann. Der Staat operiert als allgegenwärtige Norm politischer Analyse. Er ist selbstverständlich. Es wird nicht erhärtet, warum der Staat als einziger/einziges dazu in der Lage sein soll, weil diese Behauptung unter westlichem Publikum keiner Untermauerung mehr bedarf.

Der folgende Ausschnitt aus einem Interview mit einem Berater der USRegierung in Goma deutet in eine ähnliche Richtung. Er setzt den Kongo gleich zu Beginn als einen dysfunktionalen Para-Staat, um dann im zweiten Schritt das konkrete Regierungshandeln zu beschreiben. Warum er den ersten Teil benötigt, bleibt im Dunkeln.

"I think it's very difficult and you know Congo is a highly dysfunctional state given to it by the Belgians basically and reinforced strongly by Mobutu. They have a genuine para-state really. The norm of the institutions is that they find ways to make money off of the population, that's their imperative and they do not provide services, they do not act in the interest of the population, they act in their own interest to take from the population on a whole. ${ }^{8}$

Anstatt die facettenreichen Charakteristika des kongolesischen Regierungshandelns zu beschreiben, operiert dieser Analyst ausschließlich im Rahmen des pathologisierenden Verständnisprinzips Staat. Diese stellt den Kongo als gescheiterten Staat her und führt somit zum failed-state effect.

Eine bedeutende Anzahl Interviewter ist grundsätzlich bereit, dem Regierungshandeln im Kongo eine gewisse Rationalität zuzugestehen, sie erliegen aber dennoch dem Charme der Staatsnorm und stellen den Kongo als gescheiterten Staat her. Im Unterschied zur Reifizierung wird der Staat

7 Interview Donor, Goma 3, 11. September 2009.

8 Interview Donor, Goma 1, 5. Oktober 2009. 
im Modus der Gegenüberstellung nicht zu Beginn vorausgesetzt, sondern erst nachträglich nach einer grundsätzlich sachlichen Beschreibung dieser Analyse übergestülpt. In diesen Fällen steht die pathologisierende Benutzung der Staatsnorm in einem Spannungsverhältnis zu der stärker analytischen Herangehensweise an politische Prozesse im Kongo. Dass dieses Vorgehen an kognitive Dissonanz grenzen kann, zeigt folgendes Interview mit einem NGO-Manager:

KK: »What is the Congo for you«?

"A country with quite a few, I mean, an extremely fascinating, very complex country with plenty of non-... at first you think non-functioning structures, but somehow they do function but differently than you imagined. I mean, there are... it is not chaotic, there are ways of functioning and structures of power, which here... which work very well here, which are not always the ones one would like or expects. «9

Dieser NGO-Manager drückt zunächst sein deutliches Unbehagen über die dominierende pathologisierende Analytik aus. Er konzediert, dass es effektives Regierungshandeln im Kongo gibt, auch wenn es manchmal den Erwartungen der westlichen Intervent" nnen widerspricht. Dies ist ein analytisches Vorgehen. Trotz dieser Offenheit, kongolesischem Handeln eine Form von Vernunft zuzugestehen, rekurriert der Manager zu einem späteren Zeitpunkt plötzlich auf die Staatsnorm und macht den Kongo damit zu einem gescheiterten Staat. Als er über die Herausforderungen referiert, denen sich westliche Intervention im Kongo stellen muss, fragt er:

"How do I build a functioning state from this pile of cow shit? You have that in Afghanistan, in Iraq, you have it here, you have it in many of these failed states. How, how do I do statebuilding? «10

Damit werden die funktionierenden Strukturen, die manchmal der Erwartungen der westlichen Intervent*innen zuwiderlaufen, plötzlich zu einem Misthaufen, der den Kongo als gescheiterten Staat ausweist, so wie der Irak und Afghanistan. Diese pathologisierende Abkürzung zeigt wenig logische Verbindung zu dem oben Gesagtem. Dies ist eins der Kernmerkmale des Staats als Norm und Verständnisprinzip: Intervent"innen heften das failedstate-Label plötzlich an ihre Beobachtungen. Dies zeigt den Stellenwert der Staatsnorm, denn die Interviewten scheinen sich nicht gegen ihre Benut-

9 Interview NGO, Goma 2, 22. September 2009.

10 Interview NGO, Goma 2, 22. September 2009. 
zung wehren zu können, auch wenn sie es ursprünglich versucht haben mögen.

Der dritte Modus, den failed-state-effect zu produzieren, sieht den kongolesischen Staat einerseits als gescheitert oder schwach an, sucht jedoch unmittelbar nach Erklärungen für diesen Zustand, die über die ausschließliche Verantwortung der jetzigen, scheinbar unfähigen Regierung hinausgehen. Im Modus der Kontextualisierung findet eine ständige Suchbewegung nach den Ursachen für das Scheitern des Staates statt.

KK: "And what are the current main challenges for the Congo? "It's to restore or even install some state authority and then security. There are so many things that need to happen at once. Because, you know, you need to have security but you need to have a justice system but then, you know, what happens to people after they've gone through the justice system. The prisons. I mean it's just like, it's just so many things that need to happen at the same time. ${ }^{11}$

KK: "Do you have the impression that the state has no authority actually?" "No, not no authority, but it's too weak in too many places. But it has also to do with the size and the resources. And sometimes, for example, I've been to the northeast and, I don't think it's a sort of, I think sometimes it's just the geography of it. It's so far away and it will be so expensive. It hasn't been a priority. But I don't think it's something like someone sat in Kinshasa and decided, we are not gonna care about this area. It's more, you know, it's just the way it happens. " 12

Diese UN-Mitarbeiterin nimmt hier zwar einerseits den Staat und seine Autorität als selbstverständlich notwendig an, entlässt die Regierung aber teilweise aus der Verantwortung für das Fehlen des staatlichen Gewaltmonopols. Diese Herangehensweise unterscheidet sich dadurch von denjenigen der Reifizierung und der Gegenüberstellung, dass sie in einer gewissen analytischen Unsicherheit verharrt. Der Staatsnorm wird nicht automatisiert gehuldigt, sondern die Ursachen für die politische Ordnung im Kongo bleiben potenziell vielfältig und nicht zwangsläufig durch westliche Intervention behebbar.

11 Interview UN HQ, New York 5, 23. September 2011.

12 Interview UN HQ, New York 5, 23. September 2011. 


\subsection{Kongo vom Mars betrachtet}

Der obige Abschnitt hat drei verschiedene Formen aufgezeigt, den failedstate-effect unter Interventionspersonal hervorzurufen. Der Staat als Norm und Verständnisprinzip verschaffte sich während der Interviews Einlass in die Kongo-Analyse westlicher Intervent*innen, obwohl ich nicht nach dem Staat gefragt hatte. Als ich realisierte, dass der Staat einen solch überwältigenden Einfluss auf das Denken der Intervent*innen hat, entschied ich mich, eine Frage zu stellen, die Anreize dafür bot, neue analytische Pfade $\mathrm{zu}$ beschreiten. Ich versuchte, die Anreize zu freiem Assoziieren zu erhöhen, indem ich den Intervent"innen in New York und Goma folgende Frage stellte: „Stellen Sie sich vor, sie säßen auf dem Mars und ET säße neben ihnen. Sie schauen hinab auf die Erde, sie sehen, was da unten passiert. Sie sehen den Kongo und die AkteurInnen dort. Wie würden sie dies dem sehr intelligenten ET, der wie gesagt neben ihnen sitzt, beschreiben oder erklären?«

Mit dieser Frage zielte ich darauf ab, keine Terminologie zu verwenden, die die Antwort der Interviewten übermäßig stark beeinflussen könnte. Die Frage enthält die Erde, den Kongo und dortige Akteur"nnen. Dies sind die einzigen Entitäten, die ich vorschlug. Wie reagierten die Befragten nun auf diese Einladung zum Gedankenexperiment? Wie zu erwarten war, gab es eine Vielfalt an Antworten auf diese Frage. Alle der 31 Interviewten, denen ich diese Frage stellen konnte, verzichteten jedoch überraschenderweise darauf, die Begriffe Staat oder gescheiterter Staat für ihre Erklärung zu verwenden. Grundlegendere Begriffe wurden gewählt, um der Frage nach der Erde, dem Kongo und den AkteurInnen dort zu begegnen. Während dies nicht beweist, dass die Staatsnorm deplatziert ist, weist dies doch darauf hin, dass es möglich ist, über den Kongo zu reflektieren, ohne ihn zu einem gescheiterten Staat machen zu müssen.

Trotz dieser unterwarteten Staatenlosigkeit begreifen die meisten der Interviewten den Kongo dennoch als Problem. Auch in Reaktion auf die Einladung, jenseits ausgetretener Pfade zu denken, wird der Kongo vor allem pathologisiert. Es kommen jedoch neue Entitäten und Praktiken in den Blick: die menschliche Natur, die Rolle der Geschichte, die Interaktion zwischen Kollektiven im Allgemeinen oder im Kongo im Speziellen.

Für manche der Interviewten musste die Antwort auf die Marsfrage mit einer Reflexion über die menschliche Natur beginnen. Auf der einen Seite 
zeige das, was dort unten passiere, die Fähigkeit zur Gewalt ${ }^{13}$ des Menschen und es beweise, dass "everybody is able to do the best and the worst ${ }^{14}{ }^{14}$ Auf der anderen Seite könnten Menschen wählen zwischen "competition and cooperation ${ }^{15}$ im Umgang miteinander. Dieser letzte Punkt leitet über zu dem sehr prominenten Fokus auf der Interaktion zwischen menschlichen Kollektiven. Ein ehemaliger UN-Untergeneralsekretär fasste dies so:

»Well, it depends on how much political science E.T. has digested. I would probably tell E.T.: look these people over the centuries they had built social mechanisms that didn't exclude conflict but that kept conflict at a manageable level because it was conflict with wars of poor. And so even when you were the big kid on the block you were never that big. Now these people they have their own contact with the world where wealth is of different order and magnitude. And it changes the whole dynamics between power and powerlessness. Not that there was a balance in the sense of the balance that exists in democratic countries but there was a balance which was created by the limitation of means so to speak. Now, let's say, if you have control of valuable resources on the global market you have access to a level of wealth that nobody would have imagined a 100 years ago. And so your power for the good but unfortunately also for the bad increases exponentially and the stakes become much higher. And that means that the traditional mechanisms that used to sort of balance and regulate social relations are under stress. And the world of wealth thinks it can just throw in its new sophisticated structures, the rule of law, judges and courts and parliaments and all will live happily ever after. But it's not that simple. «16

Der ehemalige UN-Untergeneralsekretär erwähnt den Staat mit keinem Wort. Er bezieht sich auf Machtdynamiken und benutzt eine gradlinige Unterscheidung zwischen einer Welt des Reichtums und einer Welt der Armut. Man könnte argumentieren, dass er hier in Grundzügen eine recht klassische Modernisierungstheorie vorlegt. Die Konflikt- und Gesellschaftstheorie, die aus diesen Aussagen destilliert werden könnten, ist jedoch wesentlich globaler und ökonomischer als die dominante Perspektive des gescheiterten Staates. Meine Frage legte natürlich eine globale Perspektive auf den Kongo nahe. Im Unterschied dazu wird deutlich, wie reduziert

13 Interview NGO Goma 8, 30. September 2009, Interview NGO Goma 9, 17. September 2009.

14 Interview NGO Goma 18, 17. September 2009.

15 Interview UN HQ 8, 9. Dezember 2011.

16 Interview mit Jean-Marie Guéhenno, ehemaliger UN-Untergeneralsekretär, New York, 11. November 2011. 
und lokal die Anwendung des Staats als Verständnisprinzip im Kongo ist. Dieser Staat scheint ein Vergrößerungsglas zu sein, durch das der lokale Kontext wahrgenommen und in den Fokus gerückt und damit verständlich gemacht wird. Der Staat als Verständnisprinzip offeriert jedoch nicht den nötigen Weitwinkel, um die Szenerie in umfassenderen und grundlegenden Begriffen darzustellen.

Andere Interviewte beschrieben die Vorgänge im Kongo als einen natürlichen Konflikt der Kulturen, die es schon immer gab. Manchmal dominiere eine Kultur, manchmal eine andere. ${ }^{17}$ Ein französischer humanitärer Helfer erklärte mir den Kongo folgendermaßen:

"Ce que je lui expliquerais, c'est que dans ce monde, quelque soient les pays, il y a toujours une élite qui gouverne et qui en générale s'en met un peu plein les poches. Parmi cette élite, il y a des gens qui ont vraiment le sens de service et qui ont envie d'aider les autres, même si ils s'en mettent aussi dans la poche, et d'autres qui sont là que pour s'en mettre dans la poche et qui s'en foutent. Quand c'est le cas, il peut y avoir une conjonction d'intérêts d'entreprises multinationales de finance, de politique, de conglomérats militaire-industriels, une conjonction d'intérêts qui fait que tous ces gens-là qui ont le pouvoir vont avoir intérêt un moment à exploiter une région. Et à s'allier avec les gens locaux qui ont les mêmes. Et c'est ça, c'est ça! Je lui expliquerais un peu ça, donc tout le désastre de la période postcoloniale, c'est ça! C'est la faute à la fois des élites africaines et des élites européennes et américaines qui se sont alliées pour exploiter très clairement les richesses de l'Afrique, c'est, je veux dire c'est la criante vérité. C'est très simple. ${ }^{18}$

17 Interview, Donor Goma 2, 9. Oktober 2009.

18 Interview, NGO Goma 18, 17 September 2009, Übersetzung auf Deutsch durch den Autor: »Ich würde ET erklären, dass es in dieser Welt, egal in welchem Land, immer eine Elite gibt, die regiert und die sich die Taschen vollmacht. Innerhalb dieser Elite gibt es Leute, die einen Sinn für den Dienst am Menschen haben und die anderen wirklich helfen wollen, auch wenn sie sich dennoch die Taschen füllen. Und es gibt andere, die sich nur die Taschen voll machen und denen alles andere egal ist. Wenn letzteres der Fall ist, kann es eine Interessenallianz zwischen multinationalen Finanzunternehmen, der Politik und militärisch-industriellen Konglomeraten geben, eine Allianz der Interessen, die bewirkt, dass alle diese Leute, die die Macht haben, ein Interesse daran haben, eine Region auszubeuten. Und sich mit lokalen Leuten zu verbinden, die das gleiche Interesse haben. Das ist es, das ist es! Das würde ich ihm so ungefähr erklären, also das ganze Desaster der postkolonialen Ära, das ist es! Es ist die Verantwortung der afrikanischen, europäischen und amerikanischen Eliten, die sich zusammengetan haben, um den Reichtum Afrika auszubeuten. Das ist, möchte ich sagen, die schreiende Wahrheit. Es ist sehr einfach.« 
Erneut fehlt der Staat. Stattdessen bietet dieser humanitäre Helfer eine Analyse globaler Eliten- oder Klassenherrschaft und -ausbeutung an.

Neben diesen Theorien kollektiver Interaktion dominierten zwei weitere Aspekte die Antworten der Interviewten auf die Mars-Frage: Die Rolle der Geschichte und die Wechselbeziehung zwischen den Kongolesinnen und Kongolesen und der sogenannten internationalen Gemeinschaft.

Die Geschichte wird als eine Geschichte der Einmischung, ${ }^{19}$ des Missbrauch $s^{20}$ begriffen oder als verflucht durch den Kalten Krieg ${ }^{21}$ oder König Leopolds $^{22}$ und Mobutus Herrschaft. ${ }^{23}$ Dagegen könnten VertreterInnen des Staates als Norm- und Verständnisprinzip argumentieren, dass die Geschichte implizit in ihrer Diagnose des gescheiterten Staates enthalten ist, da seine Geschichte ein Grund für das Scheitern des kongolesischen Staates war. Es macht jedoch einen Unterschied, ob man die Geschichte in den Mittelpunkt stellt oder sich der funktionalistischen und institutionalistischen Perspektive des gescheiterten Staates bedient. Es würde beispielsweise westliche Intervent”innen zum Zögern bringen, wenn die kongolesische Geschichte als eine Geschichte konstanter Einmischung von außen begriffen würde, denn westliche Intervention schreibt genau diese Geschichte fort. Indem auf den dysfunktionalen Staat fokussiert wird, gerät diese historische Kontinuität aus dem Blick.

Die Interaktion zwischen westlichen Intervent"innen und den Kongolesinnen und Kongolesen in den Vordergrund zu stellen, macht einige interessante konzeptionelle Spannungen deutlich. Ein Angestellter der UNFriedensmission MONUSCO nimmt beispielsweise an, dass "without attention from the West, the Congo will collapse«. ${ }^{24}$ Damit artikuliert er die weitverbreitete Annahme des selbstverständlichen Nutzens westlicher Intervention. Ein Angestellter des US-State Departments argumentiert hingegen:

"The people in Congo themselves who are getting on with their lives who, and there is a lot of them, you know, there is 70 million of them now and 10 million neighbours in Rwanda and another 15 million in Uganda and more around the edges who in the day-to-day basis have direct impact on this country and on what's happening and how it's changing. And they, I think

19 Interview NGO Goma 7, 16 September 2009.

20 Interview NGO Goma 9, 17 September 2009.

21 Interview Kongolese Goma 3, 15 September 2009.

22 Interview UN HQ New York 7, 12 October 2011.

23 Interview Kongolese Goma 3, 15 September 2009.

24 Interview MONUSCO Zivilist Goma 5, 2 Oktober 2009. 
they often view all these outsiders who come and help or trying out to belp with a little, lots of scepticism. A lot of them are very smart about why the outsiders are there and how to make the most out of the outsiders being there for the period that they'll be there. So they are looking to get what they can out of it on a short term basis. «25

Ein ehemaliger kongolesischer Senator, der nun NGO-Chef in Goma geworden ist, unterstreicht diesen Punkt, als er von seiner Beziehung zu den westlichen, in diesem Fall deutschen Interventen berichtet:

"Quand je demande l'argent des allemands, c'est vrai, quand je dis aux allemands je les aime, c' est vrai puisqu' ils nous donnent de l'argent veut dire je les aime, il veut dire je vous accepte. [...] donc l'essentiel c'est de savoir, on ne peut pas ne pas faire ça pour avoir leur argent. Donc c'est tout à fait commercial et intéressé. «26

All diese Blicke vom Mars konzentrieren sich auf unterschiedliche Aspekte globalen und kongolesischen Soziallebens. Sie bieten Lesarten an, die das kongolesisch-globale Geschehen konzeptionell fassen, ohne den Staat als Norm und Verständnisprinzip zu privilegieren. Dennoch sind diese Auffassungen zu Konflikt, kollektiver Interaktion und Geschichte nicht gänzlich von der Hand zu weisen. Es würde zu interessanten analytischen Resultaten führen, wenn diese Überlegungen weiterverfolgt würden. Es ist nicht a priori entschieden, dass politische Analyse in Begriffen von Staatlichkeit und ihrem funktionalistischen Scheitern diesen Alternativen überlegen ist.

Auf einer rein logischen Ebene ist es nicht überzeugender, den Kongo als gescheiterten Staat herzustellen denn als Situation globaler Klassenkollusion oder als Ort strategischer Interaktion zwischen kongolesischen Sozialunternehmern und westlichen Gebern, die Anerkennung und Legitimität benötigen. Der Kongo ist eine multiple Realität. Der kongolesische Staat ist nur eine davon. Die Annahme, dass westliches Statebuilding das Ei des Kolumbus für den Kongo darstelle, ist auch selbst-referentieller Trend, dem westliche Interventionsakteure zu folgen beschlossen haben.

25 Interview Donor Goma 1, 5 Oktober 2009.

26 Interview NGO Goma 16, 15 September 2009. Übersetzung auf Deutsch durch den Autor: »Wenn ich die Deutschen nach Geld frage, es stimmt, wenn ich ihnen sage, dass ich sie mag, was stimmt, weil sie uns Geld geben, bedeutet ich mag euch eigentlich ich akzeptiere euch [...]. Man muss wissen, dass man das nicht machen kann, wenn man ihr Geld haben möchte. Diese Aussage ist also klar kommerziell und mit einem Hintergedanken.« 


\section{Fazit}

Der Staat war immer schon ein wichtiger Grundpfeiler der Internationalen Beziehungen und ist aktuell die zentrale konzeptionelle Basis westlicher Intervention im Kongo. In diesem Beitrag habe ich aufbauend auf einem performativen Verständnis der Praxistheorie eine Perspektive auf den Staat eingenommen, die ihn als Norm und Verständnisprinzip begreift. Indem Kongo-Intervent*innen auf den Staat zurückgreifen, stellen sie den Kongo als gescheiterten Staat her. In einem zweiten Schritt habe ich die Staatsnorm unterlaufen, indem ich gezeigt habe, dass der Kongo auch sinnvoll analysiert werden kann, ohne als gescheiterter Staat pathologisiert zu werden.

Mithilfe der drei Modi den failed-state-effect hervorzurufen - Reifizierung, Gegenüberstellung und Kontextualisierung - habe ich schließlich argumentiert, dass Intervent*innen den Staat entweder als gegeben annehmen oder ihren nuancierten Analysen kongolesischer politischer Praktiken keinen größeren Stellenwert einräumen. Diese Herangehensweisen haben den gleichen Effekt: Sie pathologisieren den Kongo und perpetuieren den failed-state-effect, der von der potentiell vielfältigen und auch vernünftigen sozialen Welt im Kongo abstrahiert. Damit legitimiert er westliche Intervention.

Wenn Intervenierende im Rahmen eines Gedankenexperimentes den Kongo vom Mars analysieren, benutzen sie plötzlich ein staatenloses Vokabular. Die Interaktion von Kollektiven, die Rolle der Geschichte und die Beziehung zwischen Kongolesinnen und Kongolesen und den Intervent*innen geraten in den Vordergrund und der Staat als Norm und Verständnisprinzip verliert an Bedeutung. Diese zwei analytischen Schritte heben hervor, dass die Staatsnorm keineswegs unentrinnbar ist.

Methodologisch betrachtet unterstreicht diese ethnographische Herangehensweise an die Herstellung des Staates unter Interventionspersonal, wie zentral ForscherInnen selbst für die Konzeption des Kongos im Rahmen der Argumentation sind. Ich habe versucht, die Fragen auf möglichst offene Weise zu stellen, sie zu interpretieren und im Zuge der Niederschrift in einen bestimmten Kontext zu setzen. Die Transparenz über meine Vorgehensweise dient dazu, meine Einbezogenheit in die Entwicklung des Arguments zu unterstreichen.

Die aktuelle praxistheoretische Debatte ermöglicht, über diskursorientiere Forschung in einem Punkt hinauszugehen: Es gelingt, das aktive Tun, auch das von Individuen, für die wiederholte Reproduktion von Praktiken und Effekten wie dem Staats- oder dem failed-state-Effekt in den Blick zu nehmen. Das Verhalten des Individuums wird nicht a priori von einer oft 
überwältigenden diskursiven Struktur vorherbestimmt, sondern Unterschiede und sich wandelnde Handlungen werden analysierbar.

Diesen theoretischen Freiheitsgewinn durch die Praxistheorie ermöglicht also ein Fokus auf die momentane Artikulation und prozesshafte Herstellung fraglicher Entitäten, wohingegen diese Entitäten oder Normen in der Normenforschung bereits gegeben sind. Durch seine argumentative Struktur wurde der Kongo in diesem Kapitel auf zwei verschiedene Arten und Weisen hergestellt - eine gescheitert, die andere staatenlos. Es mag weitere Kongos geben. Ihre Verbreitung hängt davon ab, wer was und wie viel in sie investiert. Der Kongo muss nicht auf ewig das Objekt westlichen Statebuildingverlangens bleiben.

\section{Literatur}

Abrams, Philipp 1988: Notes on the Difficulty of Studying the State, in: Journal of Historical Sociology 1: 1, 58-89.

Adler, Emanuel/Pouliot, Vincent 2011. International Practices; in: International Theory 3: 1, 1-36.

Ashley, Richard 1988: Untying the Sovereign State: A Double Reading of the Anarchy Problematique, in: Millennium - Journal of International Studies 17: 2, 227262.

Barad, Karen 2007: Meeting the Universe Halfway: Quantum Physics and the Entanglement of Matter and Meaning, Durham, NC.

Baaz, Maria E./Olsson, Ola 2011: Feeding the Horse: Unofficial Economic Activities within the Police Force in the Democratic Republic of the Congo, in: African Security 4: 4, 223-241.

Bilgin, Pinar/Morton, Andrew 2002: Historicising Representations of 'Failed States': Beyond the Cold-War Annexation of the Social Sciences?, in: Third World Quarterly 23: $1,55-80$.

Bøås, Morten/Jennings, Kathleen 2006: Insecurity and Development: The Rhetoric of the 'Failed State', in: The European Journal of Development Research 17: 3, 385-395.

Bueger, Christian/Bethke, Felix 2014: Actor-Networking the 'Failed State' - An Inquiry into the Life of Concepts, in: Journal of International Relations and Development 17: 1, 30-60.

Bueger, Christian/Gadinger, Frank 2014: International Practice Theory: New Perspectives, London.

Call, Charles 2011: Beyond the 'Failed State': Toward Conceptual Alternatives, in: European Journal of International Relations 17: 2, 303-326.

Callon, Michel 2006: What Does it Mean to Say that Economics is Performative? (CSI Working Paper Nr. 5), in: http://halshs.archives-ouvertes.fr/docs/00/09/15/9 6/PDF/WP_CSI_005.pdf; 6.8.2013. 
Campbell, David 1998: National Deconstruction: Violence, Identity and Justice in Bosnia, Minneapolis, MN.

Bliesemann de Guevara, Berit (Hrsg.) 2012: Statebuilding and State-Formation: The Political Sociology of Intervention, London.

de Olivier Sardan, Jean-Pierre 2008: Researching the Practical Norms of Real Governance, London.

Deutsches Institut für Entwicklungspolitik 2009: User's Guide on Measuring Fragility, Bonn.

Engelkamp, Stephan/Glaab, Katharina/Renner, Judith 2012: 'In der Sprechstunde'. Wie (kritische) Normenforschung ihre Stimme wiederfinden kann, in: Zeitschrift für Internationale Beziehungen 19: 2, 101-129.

Englebert, Pierre 2003: Why Congo Persists: Sovereignty, Globalization and the Violent Reproduction of a Weak State (QEH Working Paper, Nr. 95), Oxford.

Finnemore, Martha/Sikkink, Kathryn 1998: International Norm Dynamics and Political Change, in: International Organization 52: 4, 887-917.

Foucault, Michel 2006a: Sicherheit, Territorium und Bevölkerung. Geschichte der Gouvernementalität, Frankfurt/Main.

Foucault, Michel 2006b: Die Geburt der Biopolitik. Geschichte der Gouvernementalität, Frankfurt/Main.

Hansen, Thomas Blom/Stepputat, Finn 2001: Introduction, in: Hansen, Thomas/Stepputat, Finn (Hrsg.): States of Imagination: Ethnographic Explorations of the Postcolonial State, Durham, NC, 1-40.

Heathershaw, John 2012: Conclusions-Neither Built nor Formed-The Tranformation of Post-Conflict States and International Intervention, in: de Bliesemann Guevara, Berit (Hrsg.), Statebuilding and State-Formation: The Political Sociology of Intervention, London, 246-259.

Helman, Gerald/Ratner, Steven 1992: Saving Failed States, in: Foreign Policy 89, 3 20.

Herbst, Jeffrey/Mills, Greg 2009: There Is No Congo, in: Foreign Policy 18.3.2009.

Jackson, Robert/Rosberg, Carl 1982: Why Africa's Weak States Persist: The Empirical and the Juridical in Statehood, in: World Politics 35: 1, 1-24.

Keohane, Robert 1985: After Hegemony: Cooperation and Discord in the World Political Economy, Princeton, NJ.

Koddenbrock, Kai 2012: Recipes for Intervention-Western Policy Papers Imagine the Congo, in: International Peacekeeping 19: 5, 1-16.

Koddenbrock, Kai 2013: The Failed-State Effect: Statebuilding and State Stories from the Congo, in: Chandler, David/Sisk, Timothy (Hrsg.): A Routledge Handbook on Statebuilding, London, 118-129.

Koddenbrock, Kai 2014: Malevolent Politics: International Crisis Group Reporting and the Dilemmas of Rule in the DR Congo, in: Third World Quarterly 35: 4, 669-685

Koddenbrock, Kai 2015: Strategies of Critique in IR: From Foucault and Latour towards Marx, in: European Journal of International Relations 21: 2, 243-266. 
Koddenbrock, Kai 2016: The Practice of Humanitarian Intervention, London.

Koskenniemi, Martti 2005: From Apology to Utopia: The Structure of International Legal Argument, London.

Kosmatopoulos, Nikolas 2011: Toward an Anthropology of State Failure: Lebanon's Leviathan and Peace Expertise, in: Social Analysis, 55: 3, 115-142.

Law, John/Urry, John 2004: Enacting the Social, in: Economy and Society 33: 3, 390410.

MacKenzie, Donald/Muniesa, Fabian/Siu, Lucia (Hrsg.) 2007: Do Economists Make Markets? On the Performativity of Economics, Princeton, NJ.

Merlingen, Michael 2007: Everything Is Dangerous: A Critique of 'Normative Power Europe', in: Security Dialogue 38, 435-453.

Mitchell, Timothy 1991: The Limits of the State: Beyond Statist Approaches and Their Critics, American Political Science Review 85: 1, 77-96.

Mol, Annemarie 2002: The Body Multiple: Ontology in Medical Practice, Durham, NC.

Neumann, Iver 2002: Returning Practice to the Linguistic Turn: The Case of Diplomacy, in: Millennium - Journal of International Studies 31: 3, 627-651.

OECD 2011: Supporting Statebuilding in Situations of Conflict and Fragility: Policy Guidance, Paris.

Paris, Robert 2004: At War's End: Building Peace after Civil Conflict, Cambridge.

Passoth, Jan-Hendrik/Rowland, Nicholas 2010: Actor-Network State, in: International Sociology 25: 6, 818-841.

Pickering, Andrew 1994: After Representation: Science Studies in the Performative Idiom, in: PSA: Proceedings of the Biennial Meeting of the Philosophy of Science Association, 413-419.

Renders, Marleen/Terlinden, Ulf 2010: Negotiating Statehood in a Hybrid Political Order: The Case of Somaliland, in: Development and Change 41: 4, 723-746.

Schatzberg, Michael 1988: The Dialectics of Oppression in Zaire, Bloomington, IN.

Schlichte, Klaus/Wilke, Boris 2000: Der Staat und einige seiner Zeitgenossen. Zur Zukunft des Regierens in der "Dritten Welt", in: Zeitschrift für Internationale Beziehungen 7: 2, 359-384.

Sending, Ole/Neumann, Iver 2006: Governance to Governmentality: Analyzing NGOs, States, and Power, in: International Studies Quarterly 50: 3, 651-672.

Titeca, Kristof/ de Herdt, Tom 2011: Real Governance beyond the Failed State: Negotiating Education in the Democratic Republic of the Congo, in: African Affairs 110: 439, 213-231.

Trefon, Théodore 2009: Public Service Provision in a Failed State: Looking Beyond Predation in the Democratic Republic of Congo, in: Review of African Political Economy 119, 9-21.

Vrasti, Wanda 2008: The Strange Case of Ethnography and International Relations, in: Millennium - Journal of International Studies 37: 2, 279-301.

Weber, Cynthia 1991: "Writing” the State: Political Intervention and the Historical Construction of State Sovereignty (Dissertation), Phoenix, AZ. 
Weber, Cynthia 1998: Performative States, in: Millennium - Journal of International Studies 27: 1, 77-95.

Weltbank 2011: World Development Report 2011: Conflict, Security, and Development, Washington, DC.

Young, Crawford/Turner, Thomas 1985: The Rise and Decline of the Zairian State, Madison, WI.

Interviews, die im Text zitiert werden:

Donor Goma 1, 5. Oktober 2009

Donor Goma 2, 9. Oktober 2009

Donor Goma 3, 11. September 2009

Kongolese Goma 3, 15. September 2009

NGO Goma 2, 22. September 2009

NGO Goma 6, 15. September 2009

NGO Goma 7, 16. September 2009

NGO Goma 8, 30. September 2009

NGO Goma 9, 17. September 2009

NGO Goma 16, 2. Oktober 2009

NGO Goma 18, 17. September 2009

NGO Goma 19, 18. September 2009

MONUSCO Zivilist Goma 5, 2. Oktober 2009

UN HQ New York 5, 23. September 2011

UN HQ New York 7, 12. Oktober 2011

UN HQ New York 8, 9. December 2011

UN-Untergeneralsekretär, New York, 11. November 2011 
